\title{
Selective inhibition of HDAC6 sensitizes cutaneous T-cell lymphoma to PI3K inhibitors
}

\author{
MALGORZATA BOBROWICZ ${ }^{1,2}$, ALEKSANDER SLUSARCZYK ${ }^{1}$, JOANNA DOMAGALA ${ }^{1,3}$, MICHAL DWOJAK ${ }^{1,3}$, \\ DESISLAVA IGNATOVA ${ }^{2}$, YUN-TSAN $\mathrm{CHANG}^{2}, \mathrm{CHRISTOPH} \mathrm{ISELIN}^{2}$, NINA MIAZEK-ZAPALA ${ }^{1}$, \\ KATSIARYNA MARHELAVA $^{3,4}$, EMMANUELLA GUENOVA $^{2,5^{*}}$ and MAGDALENA WINIARSKA ${ }^{1^{*}}$ \\ ${ }^{1}$ Department of Immunology, Medical University of Warsaw, 02-097 Warsaw, Poland; \\ ${ }^{2}$ Department of Dermatology, University Hospital Zürich, University of Zurich, 8091 Zurich, Switzerland; \\ ${ }^{3}$ Postgraduate School of Molecular Medicine, Medical University of Warsaw, 02-097 Warsaw; ${ }^{4}$ Department \\ of Clinical Immunology, Transplantation Institute, Medical University of Warsaw, 02-006 Warsaw, Poland; \\ ${ }^{5}$ Department of Dermatology, CHUV and University of Lausanne, 1011 Lausanne, Switzerland
}

Received July 15, 2019; Accepted March 2, 2020

DOI: 10.3892/ol.2020.11587

\begin{abstract}
Histone deacetylase (HDAC) inhibitors, approved for the treatment of cutaneous T-cell lymphoma (CTCL), are non-selective agents associated with an unsatisfactory response and considerable side-effects. Targeting single HDAC isoforms is considered to provide novel therapeutic options. HDAC6 is overexpressed in primary samples from patients with CTCL and preclinical studies using transgenic mice that spontaneously develop a CTCL-like disease, have suggested that combinations including HDAC6 inhibitors may be successful in the treatment of CTCL. PI3K inhibition is currently being tested in clinical trials for CTCL with promising results. Since HDAC6 is known to diminish the activity of Akt via its deacetylation, the aim of the present study was to evaluate the therapeutic potential of selective HDAC6 inhibitors in combination with PI3K inhibitors in CTCL. Through the genetic and pharmacological inhibition of HDAC6, it was
\end{abstract}

Correspondence to: Dr Magdalena Winiarska, Department of Immunology, Medical University of Warsaw, Nielubowicza Street 5, 02-097 Warsaw, Poland

E-mail: magdalena.winiarska@wum.edu.pl

Dr Emmanuella Guenova, Department of Dermatology, CHUV and University of Lausanne, Avenue de Beaumont 29, 1011 Lausanne, Switzerland

E-mail: emmanuella.guenova@unil.ch

${ }^{*}$ Contributed equally

Abbreviations: CTCL, cutaneous T-cell lymphoma; HDAC6, histone deacetylase 6; mAbs, monoclonal antibodies; PBMC, peripheral mononuclear blood cells; PI3K, phosphoinositide 3-kinase; SS, Sézary syndrome

Key words: CTCL, HDAC6, PI3K, targeted therapy demonstrated that combining HDAC6 with PI3K inhibition may be an attractive therapeutic option for patients with CTCL.

\section{Introduction}

Cutaneous T-cell lymphoma (CTCL) is a rare malignancy of skin-homing $\mathrm{CD}^{+}$T-cells. The majority of patients suffer from non-life threatening skin symptoms that, however, significantly decrease their quality of life. In Sézary syndrome (SS), an aggressive leukemic variant of CTCL accounting for approximately $15 \%$ of CTCL cases, the clinical symptoms encompass erythroderma, lymphadenopathy, hepato-and/or splenomegaly, plantar keratoderma and alopecia (1). The prognosis is poor with overall treatment response duration rates varying from 7.5 to 22.4 months (2). Apart from standard therapies e.g., extracorporeal photopheresis (ECP), photochemotherapy, retinoids, radiation therapy, interferon (IFN)- $\alpha$, low-dose methotrexate and polychemotherapy, small-molecule inhibitors and monoclonal antibodies (mAbs) are currently being investigated for the treatment of this malignancy $(3,4)$. However, despite considerable progress being made in the management of the disease, allogeneic stem cell transplantation is currently the only curative option.

Recently, targeting epigenetic mechanisms has emerged as a novel therapeutic strategy in CTCL $(5,6)$. To date, two inhibitors of the histone deacetylase (HDAC) family, which comprises 18 enzymes with divergent functions, have been registered in the treatment of CTCL. However, the response rate to these agents remains unsatisfactory, amounting to a $30-40 \%$ overall response, followed by the acquirement of resistance (7). Moreover, one of the issues concerning the use of non-specific HDAC inhibitors are their gastrointestinal adverse effects. Thus, the development of selective inhibitors of single HDAC isoforms is considered to provide novel therapeutic options with milder side-effects (8).

It has been demonstrated that the HDAC6 isoform is overexpressed in primary samples from patients with CTCL (9) and that it is an attractive molecular target in preclinical 
experiments using a mouse model of CTCL (10). These data suggest that combination schemes including HDAC6-specific inhibitors may be successful in the treatment of CTCL. Moreover, a decrease in the intensity of pruritus, one of the main clinical issues associated with the management of CTCL, has been observed with the use of non-selective HDAC inhibitors already in use (5), as well as with the selective HDAC6 inhibitor, ricolinostat, currently tested in clinical trials on non-Hodgkin lymphoma (11).

Phosphatidylinositol 3-kinase (PI3K) inhibition is currently being tested in clinical trials on CTCL with encouraging results (12). PI3Ks are lipid kinases involved in intracellular signal transduction. In humans, four $(\alpha, \beta, \delta$ and $\gamma)$ catalytic subunits of PI3K exist. The $\delta$ and $\gamma$ isoforms are preferentially expressed in leukocytes, where they control the survival and proliferation of leukocytes. Moreover, PI3K plays a critical role in malignant transformation (13). The anti-tumor activity of PI3K inhibition in hematological malignancies relies on the blocking of survival signaling within tumor cells, as well as in non-malignant cells of the microenvironment by controlling cytokine secretion and activating the immune response $(13,14)$. Inhibitors of PI3K have already been successfully applied in the management of B-cell malignancies $(15,16)$. The results of a clinical study on T-cell lymphoma revealed an acceptable safety profile (12). Moreover, preclinical evidence of both tumor cell-autonomous and immune-mediated effects in patient-derived xenografts has been reported (12). PI3K inhibitors have already been demonstrated to synergize with the non-specific HDAC inhibitor, vorinostat (17). However, to date, at least to the best of our knowledge, no HDAC isoform responsible for this effect has been identified. As HDAC6 is known to deacetylate and to diminish the activation of Akt, a downstream kinase of the PI3K pathway (18), the aim of this study was to evaluate the therapeutic potential of selective HDAC6 inhibitors in combination with PI3K inhibitors in CTCL.

\section{Materials and methods}

Cell lines and reagents. The $\mathrm{HH}, \mathrm{H} 9$ and HUT78 cell lines were a generous gift from Dr Giandomenico Russo (Istituto Dermopatico dell'Immacolata, IDI-IRCCS, Rome, Italy). The SeAX cell line was a generous gift from Dr Katarzyna Izykowska (Institute of Human Genetics, Polish Academy of Science, Poznan, Poland). 293T cells were purchased from ATCC. The cells were maintained in RPMI-1640 medium (Invitrogen; Thermo Fisher Scientific, Inc.) supplemented with $10 \%$ fetal bovine serum, L-glutamine $(2 \mathrm{mM})$, penicillin (100 U/ml) and streptomycin $(100 \mu \mathrm{g} / \mathrm{ml})$. HDAC inhibitors (ricolinostat, citarinostat and resminostat), as well as PI3K inhibitors (duvelisib, copanlisib and pictilisib) were purchased from Selleckchem. Inhibitors were dissolved in dimethyl sulfoxide (DMSO; Sigma-Aldrich; Merck KGaA) or $10 \%$ trifluoric acid (Sigma-Aldrich; Merck KGaA) in the case of copanlisib.

Primary samples. Peripheral mononuclear blood cells (PBMCs) from patients with SS $(n=4)$ and healthy volunteers $(n=2)$ were isolated from whole blood using Histopaque 1077, according to the manufacturer's recommendations (Sigma-Aldrich; Merck KGaA). The diagnosis
Table I. sgRNA sequences used for the production of lentiviral CRISPR/Cas9 knock-out.

\begin{tabular}{lc}
\hline Sequence name & Sequence (5'-3') \\
\hline sgCON & GGGCGAGGAGCTGTTCACCG \\
sgA & CACCGAGGACACGCAGCGATCTAGG \\
sgB & CACCGCCTCTAGGATAAGGATAATG \\
\hline
\end{tabular}

of SS was established in all patients according to the World Health Organization-European Organization for Research and Treatment of Cancer criteria for SS. Blood was obtained from patients with SS at the University of Zurich Biobank (EK No. 647). This study was conducted in accordance with the principles of the Declaration of Helsinki and was approved by the Institutional Review Board of the University of Zurich (KEK-ZH-Nr. 2015-0209). Patient 3 took part in NCT02953301 clinical trial [Resminostat for Maintenance Treatment of Patients With Advanced Stage Mycosis Fungoides (MF) or SS (RESMAIN)]. Each patient signed an informed consent for the procedures.

Viability assays. Cell viability was assessed using flow cytometry or ATP activity measurement. For flow cytometry, anti-human monoclonal antibodies were used as listed: CD3 (clone BW264/56, PerCP; Miltenyi Biotec \#130-096-910), CD4 (clone VIT4; APC-Vio770; Miltenyi Biotec \#130-098-153), CD8 (clone RPA-T8, PE; BD Biosciences). The following reagents were used to discriminate dead cells: Annexin V (FITC; BD Biosciences), SYTOX (APC; Thermo Fisher Scientific) and propidium iodide (Sigma-Aldrich). For ATP activity, the Cell Titer Glo ${ }^{\circledR}$ Luminescent Cell Viability Assay (Promega) was used. Samples were acquired on a FACSCantoII (BD Biosciences). FCS Express 5 Flow Cytometry RUO, Origin Pro 9.1G and GraphPad Prism 5.0 Software were used for data analysis.

Generation of sgHDAC and lentiviral transduction. Two sgHDAC6 sequences (A and B) and sgRNA targeting green fluorescent protein (GFP) used as a control sequence $(\mathrm{CON})$ were generated with oligonucleotide pairs (Table I) and cloned into pLenti-CRISPRv2. 293T cells seeded in 6-well plates were used for the production of a replication-incompetent lentivirus. The cells were first co-transfected with $0.76 \mu \mathrm{g}$ of pLenti-CRISPRv2 and components of 2nd generation of packaging vectors, namely $0.76 \mu \mathrm{g}$ of psPAX2 and $0.5 \mu \mathrm{g}$ of pMD2.G, using standard calcium chloride method. At $48 \mathrm{~h}$ post-transfection, the lentivirusescontaining medium was collected and added to target cells at the volume ratio of 1:1. Two days later, $2 \mu \mathrm{g} / \mathrm{ml}$ puromycin was added for an additional week. Single clones were obtained from resistant cell pools by limiting dilution.

Western blot analysis. Whole-cell protein extracts were prepared by lysing cells with a buffer containing $1 \%$ Triton X-100, 50 mM HEPES (pH 7.4), 150 mM NaCl, 5 mM EDTA and $10 \%$ glycerol supplemented freshly with phosphatase and protease inhibitor cocktails. Protein concentration was measured with a Pierce BCA Protein Assay kit (Thermo Fisher Scientific, Inc.). Samples of 5-25 $\mu \mathrm{g}$ total protein 


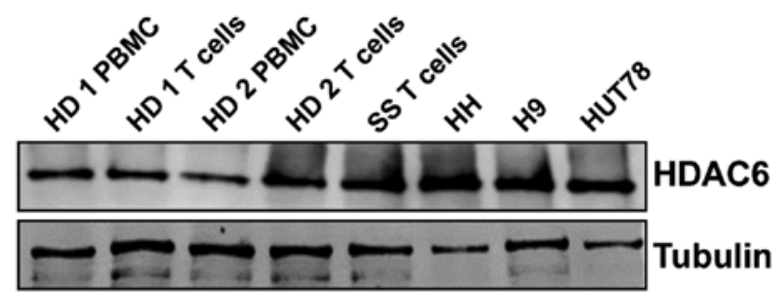

Figure 1. CTCL cell lines and primary human SS cells express HDAC6. Primary PBMCs were isolated using standard Ficoll centrifugation and further processed for the magnetic isolation of T-cells. The primary cells were incubated overnight with $100 \mathrm{IU} / \mathrm{ml} \mathrm{IL}-2 \mathrm{and} 15 \mathrm{ng} / \mathrm{ml} \mathrm{IL}-15$. Established CTCL cell lines were cultured in complete medium without the addition of cytokines. The levels of HDAC6 were assessed by western blot analysis in whole-cell lysates. Tubulin was used as a loading control. The experiments were repeated twice. HD, healthy donor; SS, Sézary syndrome; CTCL, cutaneous T-cell lymphoma; PBMCs, peripheral mononuclear blood cells; HDAC6, histone deacetylase 6.

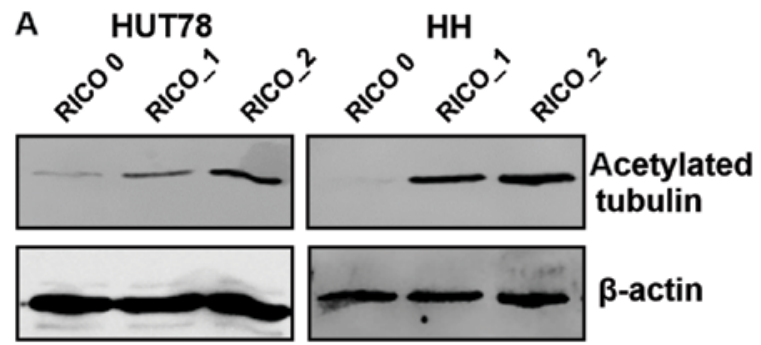

B

HUT78 Annexin V/PI

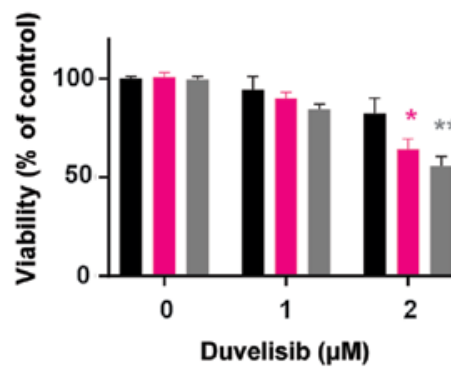

2

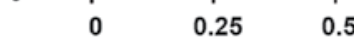

Copanlisib $(\mu \mathrm{M})$

HH Annexin V/PI
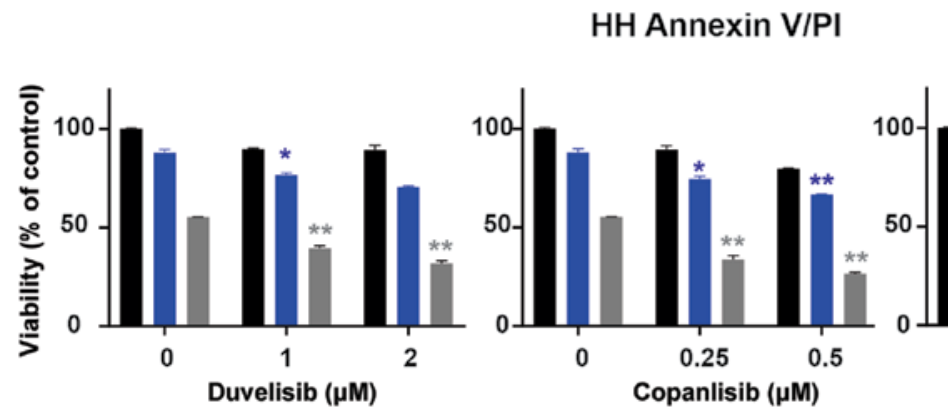

Ricolinostat ( $\mu \mathrm{M})$

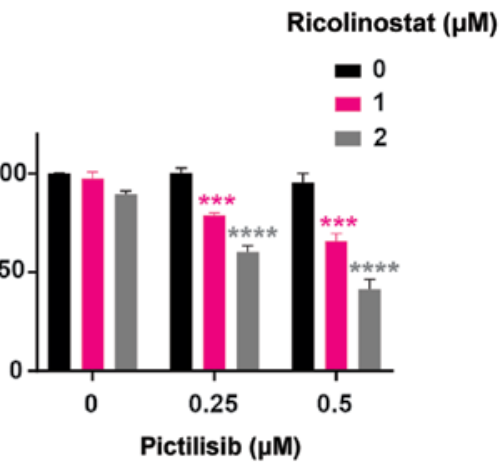

- 1

- 2

Ricolinostat $(\mu \mathrm{M})$

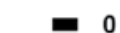

0

- 1

Figure 2. HDAC6 inhibitors sensitize CTCL cells to PI3K inhibitors. (A and B) Established CTCL cell lines (HUT78 and HH) were co-incubated for $48 \mathrm{~h}$ with increasing concentrations of the HDAC6 specific inhibitor, ricolinostat, and the following PI3K inhibitors: The non-selective inhibitor, copanlisib, the $\gamma / \delta$-selective inhibitor, duvelisib, and the pan-inhibitor pictilisib. (A) Levels of acetylated tubulin (a hallmark of HDAC6 inhibition) were assessed by western blot analysis in whole-cell lysates of HUT78 and HH cell lines incubated with 0,1 and $2 \mu \mathrm{M}$ ricolinostat (RICO_0,1,2). $\beta$-actin was used as a loading control. (B) Cell death was assessed by flow cytometry using propidium iodide or SYTOX combined with Annexin V and proliferation was assessed by ATP quantification with Cell Titer $\mathrm{Glo}^{\boxplus}$. Statistical significance was assessed using the Mann-Whitney $\mathrm{U}$ test; ${ }^{*} \mathrm{P}<0.05,{ }^{* * *} \mathrm{P}<0.01,{ }^{* * * *} \mathrm{P}<0.001,{ }^{* * * * *} \mathrm{P}<0.0001$.

were subjected to SDS-PAGE. Resolved proteins were transferred onto nitrocellulose membranes, probed with specific antibodies, and detected with Odyssey imaging system (LI-COR Biosciences). The following primary antibodies were used: Anti-HDAC6 (D2E5; Cell Signaling Technology, Inc.) and anti-acetylated tubulin (polyclonal, Sigma-Aldrich; Merck KGaA). Secondary antibodies included either HRP-conjugated antibodies from Jackson ImmunoResearch or fluorophore-conjugated (IRDye 680RD or IRDye $800 \mathrm{CW}$ ) antibodies from LI-COR Biosciences. For equal loading, the control blots were re-probed with anti- $\beta$-actin-peroxidase (AC-15, Sigma-Aldrich; Merck KGaA).

Statistical analysis. Results were plotted with GraphPad Prism. Statistical significance was assessed by appropriate tests specified in the figure legends, namely Mann-Whitney U test and 
C
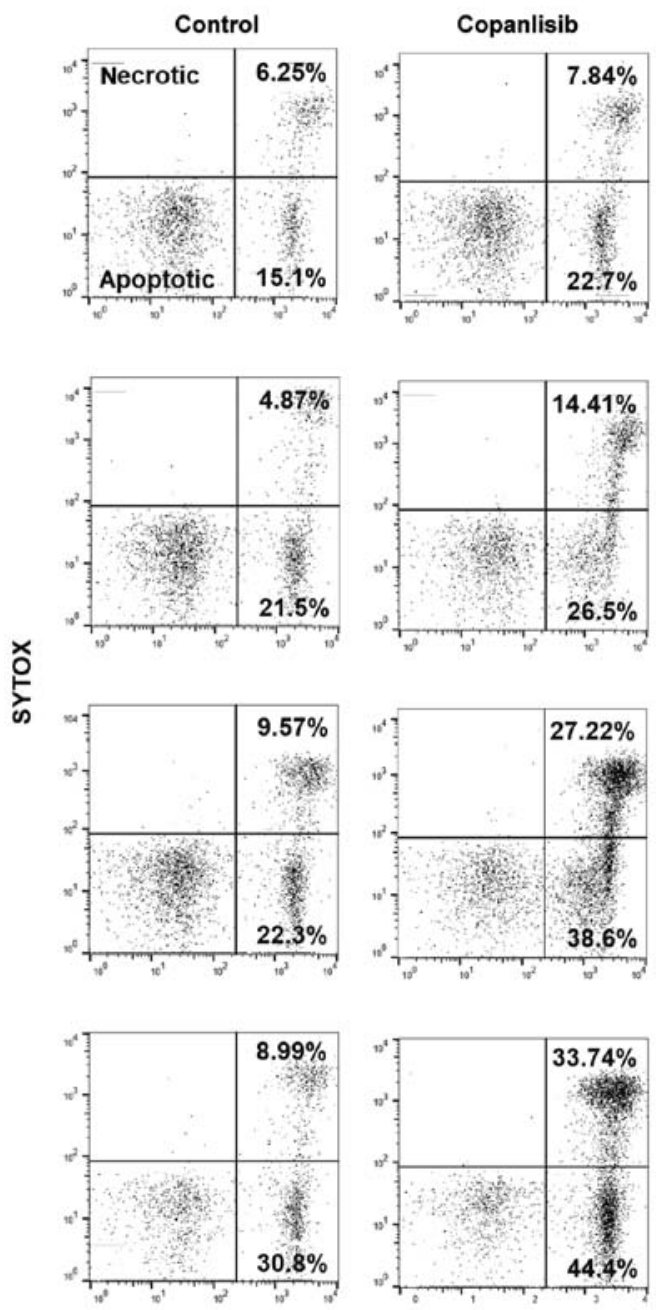

Annexin V

\section{D}

\section{Duvelisib $(\mu \mathrm{M})$}

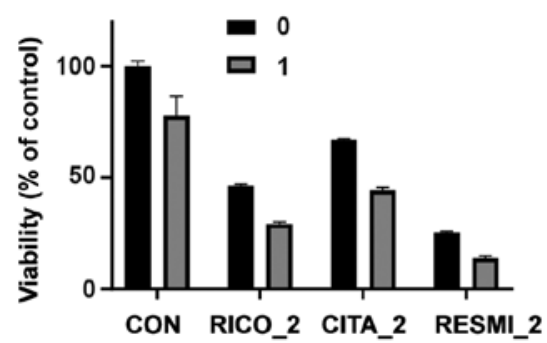

\section{Patient 1}

Copanlisib ( $\mu \mathrm{M})$

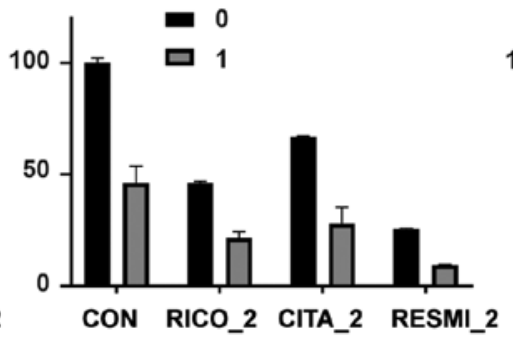

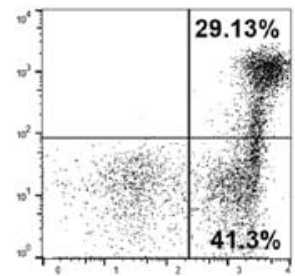

Patient 1
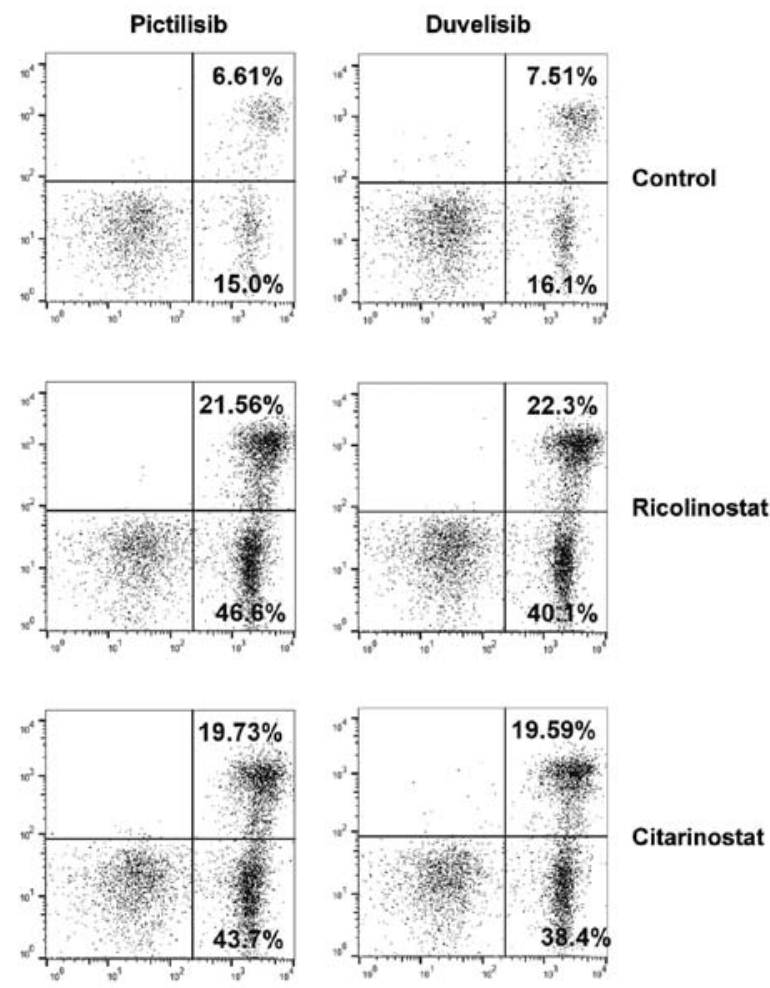

Citarinostat

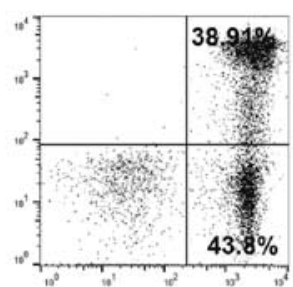

Resminostat

\section{Pictilisib $(\mu \mathrm{M})$}

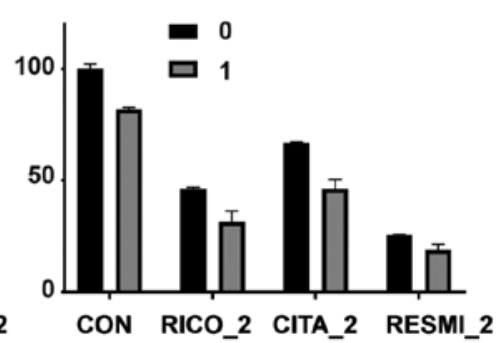

Figure 2. Continued. HDAC6 inhibitors sensitize CTCL cells to PI3K inhibitors. (C and D) HDAC6 inhibitors sensitize CTCL primary cells to PI3K inhibitors. PBMCs from a leukemic patient with CTCL were isolated using standard Ficoll centrifugation. (C) Cells were then co-incubated for $48 \mathrm{~h}$ in the presence of IL-2 and IL-15 stimulation with the HDAC 1/3/6-specific inhibitor, resminostat, or the HDAC6-specific molecules, ricolinostat and citarinostat (all at a $4 \mu \mathrm{M}$ concentration), in combination with the PI3K inhibitors, copanlisib, pictilisib and duvelisib $(1 \mu \mathrm{M})$. Cell viability was assessed by flow cytometry following Annexin V/SYTOX staining of $\mathrm{CD}^{+} \mathrm{CD}^{+}$cells. The figure shows the percentages of necrotic (upper panel) and apoptotic (lower panel) cells. (D) In addition, ATP activation was assessed using Cell Titer Glo ${ }^{\circledR}$.

two-way ANOVA with multiple comparisons with Bonferroni post-hoc test. The P-values are marked with asterisks on the charts $\left({ }^{*} \mathrm{P}<0.05,{ }^{* *} \mathrm{P}<0.01,{ }^{* * * *} \mathrm{P}<0.001,{ }^{* * * *} \mathrm{P}<0.0001\right)$. The Chou-Talalay method was used to quantify the combination index $(\mathrm{CI})$ of the tested combinations [synergistic effect $(\mathrm{CI}<1)$ ].

\section{Results}

CTCL cell lines and human primary T-cells from SS patients express HDAC6. First, the amount of HDAC6 at the mRNA and protein level was assessed in whole cell lysates of PBMCs 
E

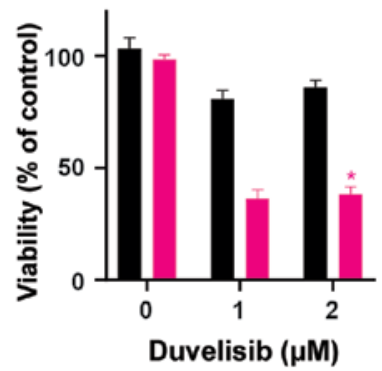

$\mathbf{F}$

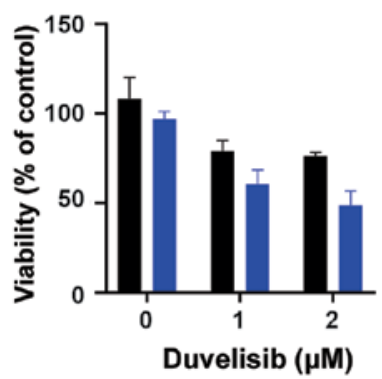

Patient 2 - non-specific HDAC inhibition
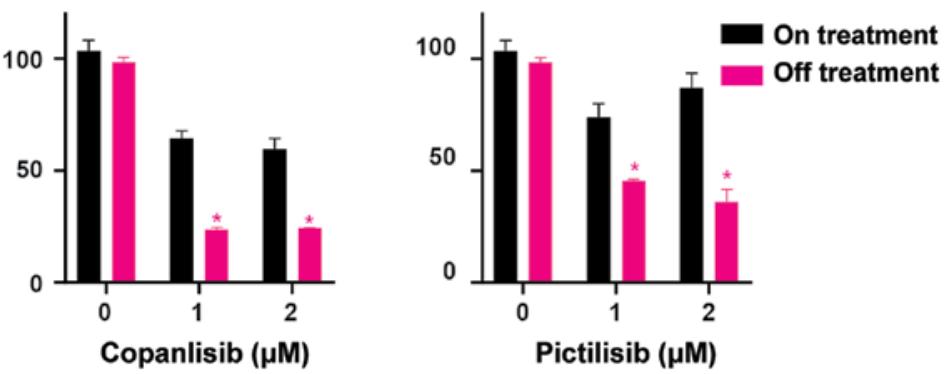

Patient 3 - specific HDAC 1/3/6 inhibition

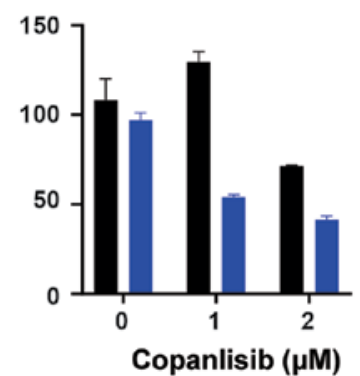

Figure 2. Continued. HDAC6 inhibitors sensitize CTCL cells to PI3K inhibitors. (E and F) HDACi-based therapy sensitizes primary CTCL cells to PI3K inhibitors. PBMCs from leukemic patients with CTCL 'on and off' therapy with (E) the HDAC pan-inhibitor, vorinostat, and (F) the HDAC1/3/6 specific inhibitor, ricolinostat, were then co-incubated for $48 \mathrm{~h}$ in the presence of IL-2 and IL-15 with 1 and $2 \mu \mathrm{M}$ PI3K inhibitors, copanlisib, pictilisib and duvelisib. Cell viability was assessed by ATP quantification using Cell Titer Glo ${ }^{\circledR}$. Statistical significance was assessed by two-way ANOVA with multiple comparisons; ${ }^{*} \mathrm{P}<0.05$. RICO, ricolinostat; CITA, citarinostat; RESMI, resminostat; CTCL, cutaneous T-cell lymphoma; HDAC6, histone deacetylase 6.

from healthy donors and T-cells, as well as in $\mathrm{CD}^{+}$cells from one patient with SS and established CTCL cell lines. The results (Fig. 1) demonstrated a strong expression of HDAC6 protein in all tested CTCL cell lines and patient samples, despite low mRNA levels (data not shown).

HDAC6 inhibitors sensitize CTCL cells to PI3K inhibitors. To evaluate the effect of HDAC6 inhibition on the sensitivity to PI3K inhibitors, the viability of two CTCL cell lines representing SS (HUT78 and HH) upon $48 \mathrm{~h}$ of incubation with the tested inhibitors was examined by flow cytometry. To block HDAC6 activity, the selective HDAC6 inhibitor, ricolinostat, was used. HDAC6 inhibition was assessed by western blot analysis (Fig. 2A). Three different PI3K inhibitors were tested: the $\delta / \gamma$-specific inhibitor, duvelisib, the pan-inhibitor, copanlisib, and the pan inhibitor, pictilisib. The results demonstrated a synergistic effect $(\mathrm{CI}<1)$ of increasing concentrations of ricolinostat in combination with all tested PI3K inhibitors in both cell lines (Fig. 2B). Moreover, in the corresponding experiments with primary T-cells isolated from patients with CTCL, a considerable, although not statistically significant potentiation of the anti-tumor activity of the tested combinations was observed, as determined by SYTOX/Annexin flow cytometry viability assay (Fig. 2C) and ATP-activity Cell Titer Glo ${ }^{\circledR}$ proliferation assay (Fig. 2D). No such effect was observed in the case of normal $\mathrm{CD}^{+} \mathrm{T}$-cells (data not shown).

Treatment with HDAC inhibitors sensitizes primary patient cells to PI3K. To assess the clinical relevance of these observations, the effects of the clinically available pan-HDAC inhibitor, vorinostat (Fig. 2E), and the clinically tested selective HDAC1/3/6 inhibitor, ricolinostat (Fig. 2F), on the efficacy of PI3K inhibition were examined. In these experiments, the in vitro sensitivity to PI3K inhibitors of primary cells isolated from patients treated in vivo with HDAC inhibitors was assessed. In the vorinostat-treated patient (Patient 2), a significant sensitization to all tested PI3K inhibitors was observed, while treatment with ricolinostat did not exert a significant effect in Patient 3. However, it should be noted that Patient 3 received ricolinostat only twice, while Patient 2 was continuing vorinostat on a regular basis.

HDAC6 knockout sensitizes the HUT78 CTCL cell line to PI3K inhibitors. In order to determine whether the observed sensitization to PI3K relies on the specific inhibition of HDAC6, HUT78 cells were stably transduced using CRISPR/Cas9 sgRNA constructs targeting HDAC6. Following puromycin selection, 2 cell lines (HUT78-sgA_HDAC6 and HUT78-sgB_ HDAC6) were obtained that were both characterized by increased levels of acetylated tubulin (a surrogate marker for HDAC6 inhibition). The HUT78-sgB_HDAC6 cells exhibited decreased HDAC6 levels and profoundly increased tubulin acetylation (Fig. 3A); thus, these cells were used to examine the efficacy of PI3K inhibitors. Some sensitization to all tested PI3K inhibitors was observed; however, the effect did not reach statistical significance (Fig. 3B). Therefore, single clones were obtained from the HUT78-sgB_HDAC6 cells by limiting dilution. Two obtained clones (sg9 and sg10) were found to exhibit HDAC6 knockout (Fig. 3C). Again, clones were tested for their sensitivity to PI3K inhibition. Both the sg9 and sg10 clones were characterized with a significantly increased sensitivity to PI3K inhibition (Fig. 3D). 

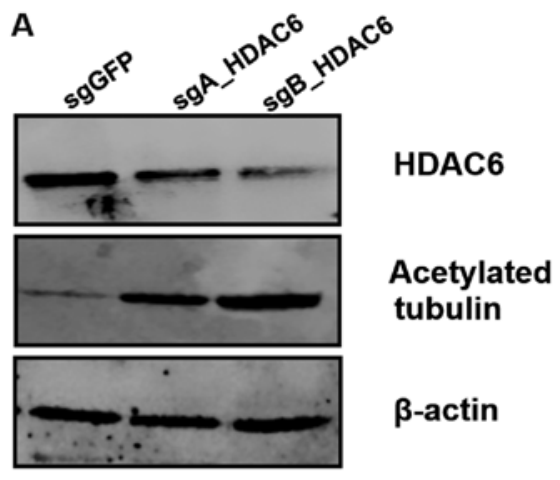

$\beta$-actin
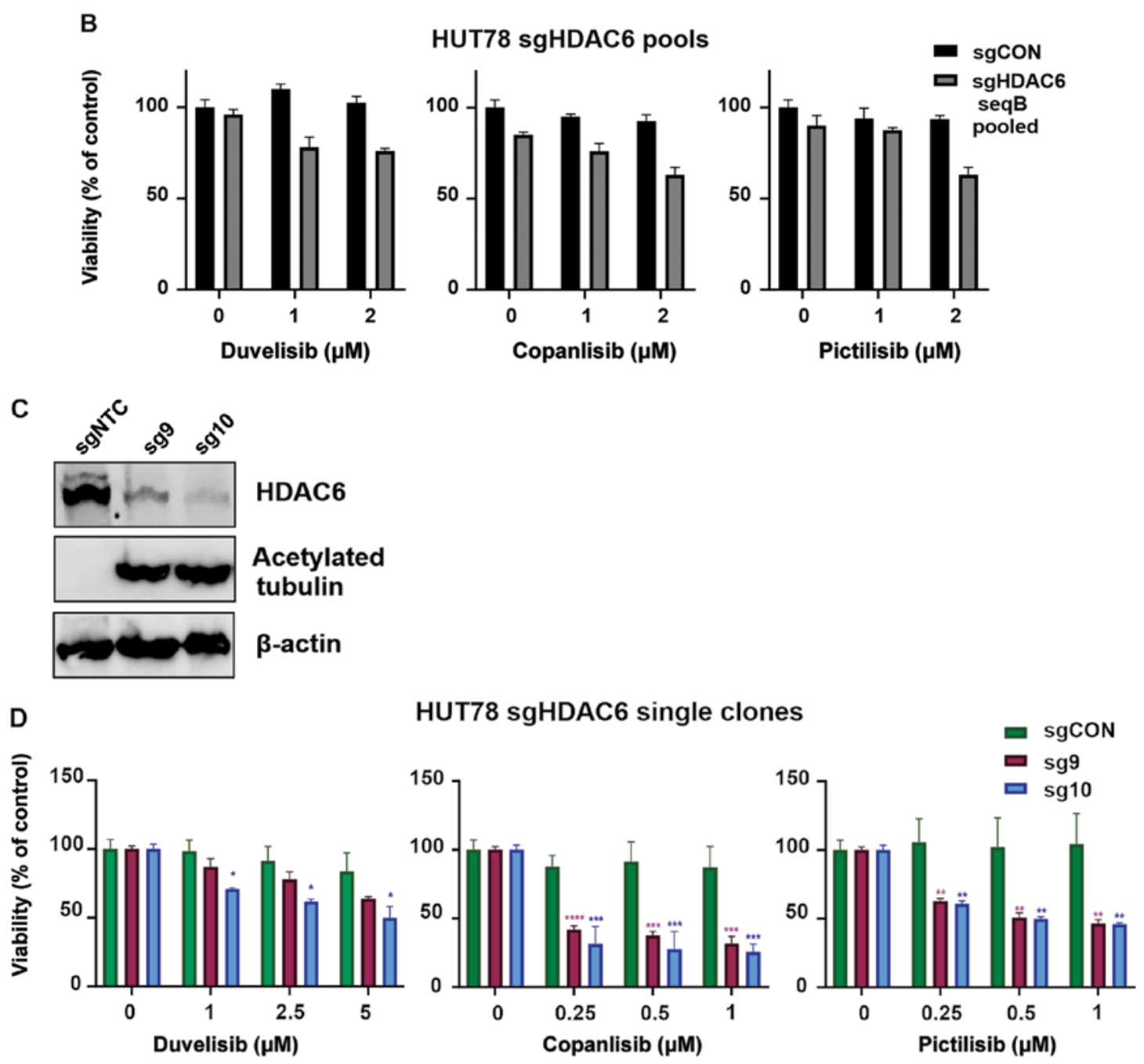

Figure 3. HDAC6 knock-out sensitizes the HUT78 CTCL cell line to PI3K inhibitors. The CTCL cell line, HUT78, was stably transduced with LentiCRISPRv.2 plasmid encoding sgRNA targeting HDAC6 (sequence A and B). sgCON was used as a non-targeting control. (A) Whole-cell lysates from pooled transduced cells were assessed for HDAC6 and acetylated tubulin (a hallmark of HDAC6 inhibition) by western blot analysis. $\beta$-actin was used as a loading control. (B) Pooled transduced cells were incubated for $48 \mathrm{~h}$ with the investigated PI3K inhibitors and their viability and proliferation were assessed with Cell Titer $\mathrm{Glo}^{\circledast}$. (C) Whole cell lysates from the cells that underwent selection with puromycin (clones sg9 and sg10) were assessed for HDAC6 and acetylated tubulin (a hallmark of HDAC6 inhibition) by western blot analysis. $\beta$-actin was used as a loading control. (D) Cells were incubated for $48 \mathrm{~h}$ with the investigated PI3K inhibitors and their viability and proliferation assessed with Cell Titer Glo ${ }^{\circledR}$. Statistical significance was assessed by two-way ANOVA with a Bonferroni post-hoc test. ${ }^{*} \mathrm{P}<0.05,{ }^{* *} \mathrm{P}<0.01,{ }^{* * *} \mathrm{P}<0.001,{ }^{* * * *} \mathrm{P}<0.0001$ vs. $\operatorname{sgCON}$. CTCL, cutaneous T-cell lymphoma; HDAC6, histone deacetylase 6 ; sgCON, Sg sequence targeting GFP.

\section{Discussion}

SS represents an aggressive leukemic variant of CTCL with a poor prognosis. Despite their established position in the treatment of CTCL, HDAC inhibitors do not lead to durable remissions. However, preclinical data suggest the potential role of HDAC inhibitors in combination treatment. As the pan-HDACi inhibitor, vorinostat, has already been demonstrated to sensitize CTCL to PI3K inhibition, this study examined whether this mode of action may result from the 
inhibition of a single HDAC isoform, HDAC6. HDAC6 is an isoform shown to be overexpressed in CTCL and a druggable target with demonstrated preclinical efficacy in B-cell malignancies. HDAC6 inhibition using its small-molecule inhibitor, ricolinostat, has been shown to exert a direct tumor-killing effect, as well as to sensitize malignant cells to a variety of drugs with different mechanisms of action $(11,19,20)$. Importantly, data from clinical trials suggest its improved safety profile when compared to non-specific HDAC inhibitors (21). PI3K inhibition has recently been proposed as a novel treatment option for CTCL. In this study, it was demonstrated that the anti-tumor effects of PI3K inhibitors can be further potentiated by HDAC6 inhibition. In this study setting, a synergistic effect of HDAC6 inhibition was observed, combined with three different both isoform-specific, as well as pan-PI3K inhibitors on CTCL established cell lines. Moreover, an additive effect was observed on primary $\mathrm{CD}^{+}$cells obtained from patients with SS, that are regularly observed to be apoptosis-resistant. In this study, using both pharmacological (HDAC6-selective inhibitor), as well as genetic tools (CRISRP/Cas9 system) it was demonstrated that the observed sensitization to PI3K inhibition relies on HDAC6 blockage. Although the mechanistic basis of this phenomenon requires additional functional experiments, the combination of PI3K with HDAC6 inhibitors seems to be a rational approach with which to enhance the cytotoxic effects in CTCL. The results of this study contribute to the opening of new avenues for the use of specific HDAC-inhibitors in CTCL (22). To date, the expression of HDAC2 has been linked to a more aggressive phenotype of CTCL (9). In addition, the selective targeting of HDAC3 may be an option for CTCL, as it has been shown to disrupt the DNA replication of rapidly cycling CTCL cells (23). Moreover, quisinostat, an inhibitor with the highest potency to inhibit HDAC1, has demonstrated encouraging results in modifying the phenotype of the HUT78 cell line (24). Therefore, inhibitors of class I HDACs seem to be promising in this type of malignancy. Indeed, domatinostat (4SC-202), a novel inhibitor of class I HDACs, has been demonstrated to effectively inhibit the growth of CTCL cells (25). It is anticipated that in the future, novel isoform-specific compounds will emerge.

\section{Acknowledgements}

This abstract was presented at the EORTC Cutaneous Lymphoma Task Force Meeting 2018 (September 27, 2018-September 29, 2018; St. Gallen, Switzerland), and was published as Abstract no. 025.

\section{Funding}

The present study was supported by the European Commission Horizon 2020 Program 692180STREAMH2020-TWINN-2015 (to MB, JS, MD and MW), the Polish National Science Centre 2015/18/E/NZ6/00702 (to MB, JS and MW), EMBO (short-term fellowship no. 7637 to $\mathrm{MB}$ ), the Ministry of Science and Higher Education (2019/94/DIR/NN3 to MB) the Promedica Stiftung (1406/M and $1412 / \mathrm{M}$, both to EG), the Swiss Cancer Research Foundation (KFS-4243-08-2017 to EG) and the Clinical Research Priority Program (CRPP) of the University of Zurich (to EG). The funders had no role in the study design, data collection and analysis, decision to publish, or preparation of the manuscript.

\section{Availability of data and materials}

The datasets used and/or analyzed during the current study are available from the corresponding author on reasonable request.

\section{Authors' contributions}

JD, MD, DI, YTC, CI, AS and KM performed the experiments and analyzed the data. MB and NMZ performed the experiments and analyzed the data, and were involved in the manuscript preparation. EG and MW contributed to the conception and design of the study, were involved in drafting the manuscript and data interpretation, and provided expert advice and guidance throughout the study. All authors read and approved the final manuscript.

\section{Ethics approval and consent to participate}

The present study was conducted in accordance with the principles of the Declaration of Helsinki and approved by the Institutional Review Board of the University of Zurich (KEK-ZH-Nr. 2015-0209). Each patient provided written informed consent for the procedures.

\section{Patient consent for publication}

The patients provided consent for the publication of the results.

\section{Competing interests}

The authors declare that they have no competing interests.

\section{References}

1. Mangold AR, Thompson AK, Davis MD, Saulite I, Cozzio A, Guenova E, Hodak E, Amitay-Laish I, Pujol RM, Pittelkow MR and Gniadecki R: Early clinical manifestations of Sezary syndrome: A multicenter retrospective cohort study. J Am Acad Dermatol 77: 719-727, 2017.

2. Janiga J, Kentley J, Nabhan C and Abdulla F: Current systemic therapeutic options for advanced mycosis fungoides and Sezary syndrome. Leuk Lymphoma 59: 562-577, 2018.

3. Guenova E, Hoetzenecker W, Rozati S, Levesque MP, Dummer R and Cozzio A: Novel therapies for cutaneous T-cell lymphoma: What does the future hold? Expert Opin Investig Drugs 23: 457-467, 2014.

4. Saulite I, Hoetzenecker W, Weidinger S, Cozzio A, Guenova E and Wehkamp U: Sezary syndrome and atopic dermatitis: Comparison of immunological aspects and targets. Biomed Res Int 2016: $9717530,2016$.

5. Moskowitz AJ and Horwitz SM: Targeting histone deacetylases in T-cell lymphoma. Leuk Lymphoma 58: 1306-1319, 2017.

6. Quaglino P, Maule M, Prince HM, Porcu P, Horwitz S, Duvic M, Talpur R, Vermeer M, Bagot M, Guitart J, et al: Global patterns of care in advanced stage mycosis fungoides/Sezary syndrome: A multicenter retrospective follow-up study from the cutaneous lymphoma international consortium. Ann Oncol 30: 494, 2019.

7. Wilcox RA: Cutaneous T-cell lymphoma: 2017 update on diagnosis, risk-stratification, and management. Am J Hematol 92: 1085-1102, 2017.

8. West AC and Johnstone RW: New and emerging HDAC inhibitors for cancer treatment. J Clin Invest 124: 30-39, 2014. 
9. Marquard L, Gjerdrum LM, Christensen IJ, Jensen PB, Sehested M and Ralfkiaer E: Prognostic significance of the therapeutic targets histone deacetylase 1, 2,6 and acetylated histone H4 in cutaneous T-cell lymphoma. Histopathology 53: 267-277, 2008

10. Mishra A, La Perle K, Kwiatkowski S, Sullivan LA, Sams GH, Johns J, Curphey DP, Wen J, McConnell K, Qi J, et al: Mechanism, consequences, and therapeutic targeting of abnormal IL15 signaling in cutaneous T-cell lymphoma. Cancer Discov 6: 986-1005, 2016.

11. Amengual JE, Prabhu SA, Lombardo M, Zullo K, Johannet PM, Gonzalez Y, Scotto L, Serrano XJ, Wei Y, Duong J, et al: Mechanisms of acquired drug resistance to the HDAC6 selective inhibitor ricolinostat reveals rational drug-drug combination with ibrutinib. Clin Cancer Res 23: 3084-3096, 2017.

12. Horwitz SM, Koch R, Porcu P, Oki Y, Moskowitz A, Perez M, Myskowski P, Officer A, Jaffe JD, Morrow SN, et al: Activity of the PI3K- $\delta, \gamma$ inhibitor duvelisib in a phase 1 trial and preclinical models of T-cell lymphoma. Blood 131: 888-898, 2018.

13. Vanhaesebroeck B, Guillermet-Guibert J, Graupera M and Bilanges B: The emerging mechanisms of isoform-specific PI3K signalling. Nat Rev Mol Cell Biol 11: 329-341, 2010.

14. Kaneda MM, Messer KS, Ralainirina N, Li H, Leem CJ, Gorjestani S, Woo G, Nguyen AV, Figueiredo CC, Foubert P, et al $\mathrm{PI} 3 \mathrm{~K} \gamma$ is a molecular switch that controls immune suppression. Nature 539: 437-442, 2016.

15. Balakrishnan K, Peluso M, Fu M, Rosin NY, Burger JA Wierda WG, Keating MJ, Faia K, O'Brien S, Kutok JL and Gandhi V: The phosphoinositide-3-kinase (PI3K)-delta and gamma inhibitor, IPI-145 (Duvelisib), overcomes signals from the PI3K/AKT/S6 pathway and promotes apoptosis in CLL. Leukemia 29: 1811-1822, 2015.

16. Flinn IW, Hillmen P, Montillo M, Nagy Z, Illes A, Etienne G, Delgado J, Kuss BJ, Tam CS, Gasztonyi Z, et al: The phase 3 DUO trial: Duvelisib vs ofatumumab in relapsed and refractory CLL/SLL. Blood 132: 2446-2455, 2018.

17. Wozniak MB, Villuendas R, Bischoff JR, Aparicio CB, Martinez Leal JF, de La Cueva P, Rodriguez ME, Herreros B, Martin-Perez D, Longo MI, et al: Vorinostat interferes with the signaling transduction pathway of T-cell receptor and synergizes with phosphoinositide-3 kinase inhibitors in cutaneous T-cell lymphoma. Haematologica 95: 613-621, 2010.
18. Iaconelli J, Lalonde J, Watmuff B, Liu B, Mazitschek R, Haggarty SJ and Karmacharya R: Lysine deacetylation by HDAC6 regulates the kinase activity of AKT in human neural progenitor cells. ACS Chemical Biology 12: 2139-2148, 2017.

19. Cosenza M, Civallero M, Marcheselli L, Sacchi S and Pozzi S: Ricolinostat, a selective HDAC6 inhibitor, shows anti-lymphoma cell activity alone and in combination with bendamustine. Apoptosis 22: 827-840, 2017.

20. Bobrowicz M,Dwojak M,Pyrzynska B,Stachura J, Muchowicz A, Berthel E, Dalla-Venezia N, Kozikowski M, Siernicka M, Miazek N, et al: HDAC6 inhibition upregulates CD20 levels and increases the efficacy of anti-CD20 monoclonal antibodies. Blood 130: 1628-1638, 2017.

21. Vogl DT, Raje N, Jagannath S, Richardson P, Hari P, Orlowski R, Supko JG, Tamang D, Yang M, Jones SS, et al: Ricolinostat, the first selective histone deacetylase 6 inhibitor, in combination with bortezomib and dexamethasone for relapsed or refractory multiple myeloma. Clin Cancer Res 23: 3307-3315, 2017.

22. Lopez AT, Bates S and Geskin L: Current status of HDAC inhibitors in cutaneous T-cell lymphoma. Am J Clin Dermatol 19: 805-819, 2018.

23. Wells CE, Bhaskara S, Stengel KR, Zhao Y, Sirbu B, Chagot B, Cortez D, Khabele D, Chazin WJ, Cooper A, et al: Inhibition of histone deacetylase 3 causes replication stress in cutaneous $\mathrm{T}$ cell lymphoma. PLoS One 8: e68915, 2013.

24. Mehul B, Perrin A, Grisendi K, Galindo AN, Dayon L, Menigot C, Rival Y and Voegel JJ: Mass spectrometry and DigiWest technology emphasize protein acetylation profile from quisinostat-treated HuT78 CTCL cell line. J Proteomics 187: $126-143,2018$.

25. Wobser M, Weber A, Glunz A, Tauch S, Seitz K, Butelmann T, Hesbacher S, Goebeler M, Bartz R, Kohlhof H, et al: Elucidating the mechanism of action of domatinostat (4SC-202) in cutaneous T cell lymphoma cells. J Hematol Oncol 12: 30, 2019.

This work is licensed under a Creative Common Attribution-NonCommercial-NoDerivatives 4.0 International (CC BY-NC-ND 4.0) License. 